\title{
Review of Present Trends and Future Scope of Pharmacogenomics in Drug Discovery and Development Process
}

\author{
V.Chandrakala ${ }^{1 *}$, Utpal Kumar Sanki ${ }^{2}$, \\ ${ }^{1}$ East Point College of Pharmacy, Bidrahalli, Bangalore-560049, India \\ ${ }^{2}$ Novotech International Ltd, Bangalore-560001, India.
}

\begin{abstract}
Pharmacogenomics combines traditional pharmaceutical sciences such as biochemistry with annotated knowledge of genetics, protein chemistry, and DNA polymorphisms. The difference of therapeutic efficacy of the same drug in different individual can be best explained by the study of genetic polymorphisms that underlie individual differences in drug response. The small change of the genome in one individual may make a drug inefficacious as oppose to the other patients. Such variability will bring the individualization of the therapy to obtain the best effects of the drug, as an example autologous dendrimer got a tremendous success in the cancer therapy of the individual.

Markers of exposure can determine whether the desired target tissues of a subject have been exposed to a drug at physiological concentrations. Gene expression profiling is a tool that can be used to characterize chemically induced toxicity in cells and/or animal models, in order to provide plausible explanations for observed toxicity in preclinical testing.

Pharmacogenomics holds the promise that drugs might one day in future be tailor-made for individuals and adapted to each person's own genetic makeup by the use of microarray technology to express the gene which in turn helps to develop receptor protein. The stability of the drug and its toxicity can be predicted prior to administration of the drug to the subject through the knowledge of computer assisted structural simulation and DNA reactivity technology respectively.

This article provides some critical aspects of drug developments, clinical trial design and ethical issues which are centered with pharmacogenomics.
\end{abstract}

Keywords : Pharmacogenomics, Gene Expression, Autologous Dendrimers, genetic polymorphism.

\section{Introduction}

Pharmacogenomics is the study of the genetic makeup of an individual in the drug response. It is concerned with developing drug therapies to compensate for genetic differences in patients which cause varied 
responses to a single therapeutic agent. Drug -disease and patient-drug interactions are complex trait influenced by many genes. There are few genes individually or in a group alter the therapeutic repose of a drug. Diversity of genetic organization in a population, it is hardly possible to develop genetic tests which predict individual drug response. Pharmacogenomics is a science that examines the inherited variations in genes that dictate drug response and explores the ways these variations can be used to predict the kind of response a patient would have to a drug.

Pharmacogenomics refers to the entire sets of genes that determine pharmacodynamics and sometime pharmacokinetics of the drug whereas pharmacogenetics often refers to subset of the gene inherited by an individual often resulted in difference in drug metabolism and disposition. Since this distinction is capricious, these two terms are now commonly used as a reciprocal to each other. As summarized by World Health Organization (WHO) Drug Information, pharmacogenetics refers to the study of DNA sequence variations among individuals relating to differential drug response, i.e. the use of genomics to determine an individual's pharmacodynamics response towards drugs and pharmacogenomics utilizes nucleotide sequences of an individual for drug targeting to the specific organ or specific genomic population (1).

The benefits of pharmacogenomics are huge and diverse. Clinical research organization could use genotyping to exclude those patients having different drug effects than expected, from the clinical trials. This obviously potentiate the therapeutic efficacy of a drug to a particular population which is otherwise ineffective. Pre-screening the clinical trial subjects also helps in reducing the drug costs by allowing smaller clinical trial at faster rate with reduce cost (2).

Knowledge of individual genetic makeup helps physician to prescribe better drugs or alternative therapy to build his confidence as well as patient's satisfaction (3). However, while pharmacogenomics provides an appealing alternative to medications that have fewer side effects, individualization in drug therapy in this way is not as simplistic as it appears.

Heterogeneity of disease progress and the individual response to drugs vary and so it should be expected that with all therapeutics some patients respond, some do not and some have an exaggerated response. The source of individual variation in response to drugs may be single nucleotide polymorphisms (SNPs) or mutation. Human genome are rich of SNPs, may affect the pharmacokinetics and pharmacodynamics of a drug. Genetic mapping or genomic expression studies are playing pivotal role to express one's cell, tissues, organ or organ systems function. Since numerous genes are involved in controlling single function, such researches are always compromised to identify correct gene responsible for disease progression, drug targeting, markers, diagnostics and relates drug toxicity.

Allelic variations in the drug-metabolizing cytochrome P450s group of enzymes have significant effects on drug metabolism. Expression of such genes in human are regulated by toxic gases exposure, endogenous \& exogenous hormones therapy, exposure to radiations, indiscriminate use of drugs $\&$ xenobiotics, nuclear and DNA alternations $(4,5)$. All these preliminary works on genetic research are evidence of beginning of endless pharmacogenomics drug development era.

\section{Need for Pharmacogenomics}

Pharmacogenomics offers a wide area for research. Polymorphism of genes coded for drug metabolizing enzymes, specifically those belonging to cytochrome P450's family may lead to alteration of drug delivery, development and therapeutic efficacy of drugs. Genotyping being costly approach for drug development once are now affordable with an advanced method and incorporation to the patient's record can be used for the guidance of the physician in individualizing the treatment (6). Not only pharmacogenetics and pharmacoproteomics, pharmacogenomics also represents the advent of personalized medicine designed to match a treatment for a patient according to his genotype. As genotyping is not a clinically accepted technique currently due to its failure at many situations, more work is needed to rationalize this treatment options for the betterment of the society. Individualization of treatment with the help of pharmacogenomics claimed improvised therapeutic benefit of the treatment with a reduced cost. However, some ethical, social and racial issues need to be addressed as per FDA guidelines on pharmacogenomics before implementing such therapy to the society. Apart commercialization of personalized medicines against conventional blockbuster molecule limits it's further implementation to the society. High level of accuracy and precession is necessary for the clinical testing needed by an individual in order to proceed with individualized drug therapy $(7,8)$. Current 
results in the field of pharmacogenomics have not been robust, but with the advent of high throughput screening, bigdata analysis techniques with the help of computer aided drug designing techniques will significantly improve pharmacogenomics drug development. Drug development in future is expected to have genomic approaches, a future promise of pharmacogenomics.

\section{What Recent Research Promises}

Current pharmacogenomics researches are oriented with how alliances with niche technology providers can boost the commercial potential of the abundance of targets available in future genetic research. Numerous techniques have been combined to identify drug resistance gene for tuberculosis, HIV infections, cancer chemotherapy, schizophrenia, antidepressant therapy, anti-hyperlipidemia therapy, heart disease management, asthma management, nervous system disorders etc, so that personalized medicine can be engineered as per therapeutic need. Pharmaceutical companies are suffering to potentiate sale force for pharmacogenomics derived products due to lack of market segment for such niche sector medicament. In recent days pharma companies are concentrating on generating high volume sale by offering portfolios of best in therapy and multiple therapy pharmacogenomics-derived products out of conventional block buster molecules. With the advancement of technology pharmacogenomics-derived products offers a broad range of therapy options with uncompromised safety thereby increasing the productivity of R\&D otherwise sinking due to lack of innovations (9).

\section{Pharmacogenomics in Drug Development}

There was a strong believe that the Human Genome Project could reveal many surprising targets against which new therapy could be revolutionized. Unfortunately, this promise is yet to be realized, there are still many areas of human genome and disease progression has been untouched upon Human Genome Project. However, it revealed many undiscovered facts about human genome to yield new insights into the pathogenesis of human diseases and to reveal new strategies for their prevention or treatment. In depth knowledge of Pharmacogenomics will increase the chances of drug discovery and development in many ways specially, by identifying novel targets, metabolizing enzyme, disease progression route, resistant gene and by subpopulationspecific drug development. For example, genomics information can be utilized to identify, mutated gene responsible for rapid drug disposition \& metabolism, differentiate resistant gene which are to be considered as novel targets for future drug designing. For instance, cancer cells are prone to mutate faster than normal cell, hence an anticancer agent which is effective at initial state may be ineffective at later stage of cancer due to over or under expression of mutated gene. These mutated genes represent potential targets for inhibitors that could reverse the drug resistance phenotype. Other applications includes gene expression in target tissues (for example, tumor cells) to ascertain the efficacy of a single or combine chemotherapeutic agents, identifying genetic polymorphisms that determine adverse drug effects to a small subset of the population treated with a new medication, could jeopardize further development of the drug for all patients. One promising technique could be obtaining genomic DNA from patients entered on large Phase III clinical trials of a new agent, and retrospectively search for genetic polymorphisms in that subset of patients who developed toxicities or who responds to the drug therapeutically. The ultimate goal of the new therapeutic agents under development should have uncompromised benefits with no or less toxicity and which are only possible by the use of genetic information from the patient's clinical database (10)

Pharmacogenetics could be incorporated into clinical development programs so that efficacy of medicine response profiles (MRPs) could be generated from patient's DNA testing. DNA data from the phase I trial will help to identify specific marker involved in therapeutic response of the drug, by doing so we can enrich phase III trial. By collecting DNA samples in Phase II clinical trials and identifying DNA markers that correlate with defined efficacy parameters, it might be possible to further focus Phase III clinical trials by recruiting only those patients likely to respond. This will make these studies more efficient. In addition, information collected that identifies 'non-responders' in Phase II studies could be used in drug discovery to find new drugs to meet current unmet need in real time, rather than many years after trial-and-error post marketing. In fact, using genetic and genomic technologies to improve our understanding of variations in a drug molecule's metabolic profile or target before it enters phase II clinical trials will decrease overall attrition rates. Interestingly, one of the major points of failure in drug development is preclinical toxicology. Regulatory toxicology studies add at least two years to the drug development programs. Designing experiments that provide predictive toxicology information in 1-2 months rather than 1-2 years could increase the efficiency by 
better utilization of resources that are currently used for longer toxicology studies. A greater throughput of NCEs that are less likely to fail regulated toxicology studies could lower the overall cost of drug development. This greater efficiency will provide opportunities to target diseases that are currently thought to be providing a low level of return on research and development investment.

Some companies are trying to introduce pharmacogenetic evaluations from the very early stages of drug development, that is from phase I and phase II studies. The aim is to develop pharmacogenetic tests, when this is desirable and feasible, alongside drug development to reduce attrition in the pipeline and then guide clinical practice. Pharmacogenomics can provide new targets from the study of genes involved in diseases. From the knowledge of gene function and of their role in the disease pathway, new targets can be derived, that might be innovative and not accessible with non-genomic approaches (11).

\section{Challenges Posed by Pharmacogenomics}

The challenges to the industry created by pharmacogenetics and pharmacogenomics are significant and include the following:

- Genotyping will identify many new disease-related genes and provide an explosion of new targets to pursue

- Pharmacogenomics profiling will lead to patient stratification, and these new targets, as well as existing targets, will be divided into subsets.

It is estimated that genotyping will identify new disease related genes that will lead to between 5,000 and 10,000 new potential targets. Because the current amount of targets is approximately 503 comprised of mainly four target classes, such as G-protein-coupled receptors (GPCRs), ion channels, nuclear hormone receptors and enzymes, these new targets will add genomic and medicinal diversity (12).

\subsection{Scientific hurdles}

Post-genomic science guarantees a 10-fold increase in the number of drug targets but target validation bottlenecks must be overcome. Pharmacogenomic processes remain difficult owing to population-specific sources of error, limits of experimental approaches, and the challenges of investigating the many potential regulatory modes, including accounting for epistasis and epigenetics. In some cases, if well-characterized genes are indicative, a few common polymorphisms within a population will cover the bulk of the genetic variation while many, less frequent polymorphisms will explain the rest $(13,14)$.

Consider, for instance, using pharmacogenomic approaches to cardiovascular diseases. The everyday reality of scientific research brings in several problems :

$>$ The definitions of cardiovascular diseases, which allow the distinction between normal and pathologic, are founded on heterogeneous clinical and biologic bases.

$>$ The transcription analysis techniques are not standardized, and the studies of reproducibility limits the generalizations of the conclusions drawn.

$>$ Multiple differences are normally observed, and isolation of several transcribed sequences is not an easy task.

\subsection{Commercial hurdles-}

The commercial hurdles encountered by pharmacogenomics at the drug developmental and marketing stages are:

\subsubsection{In the developmental stage -}

Micro-segmented markets do not necessarily mean reduced revenue streams for pharmacogenomicsderived products because genotype screening identifies areas of high unmet need. In fact, the application of pharmacogenomics in cancer significantly increases the revenue potential of drugs with narrow therapeutic indices. 
An important factor to consider is that despite the promise of more efficient markets, and smaller stage trials which cost less, pre-clinical and early- stage development costs may increase as a result of the cost of designing gene-base assays and then using these assays to screen and select patients for inclusion in later clinical trials. Essentially, this represents a shift in the stages of drug development which might prove to be a problem for smaller companies. Also, market segmentation would result in the decrease in the revenue. However, some pharmacogenomics-based drugs still become blockbuster drugs, as the breast cancer therapy trastuzumab aptly demonstrates (15).

\subsubsection{In the marketing stage}

Traditional mass marketing is ineffective at positioning pharmacogenomic interventions and to achieve this, radical new approaches are required. Regulatory bodies would require a diagnostic test before pharmacogenomics-derived products are approved. Maximizing the commercial potential of pharmacogenomics-derived products through effective targeting of patients, physicians and identifying the clinical trial stages that benefit most from integrating pharmacogenomics, targeting therapeutic markets effectively by applying genomic-based technologies to improve pipeline quality, reducing time and costs by learning from the industry's leaders when optimizing the commercial potential of genomics-based development strategies.

The impact of genomic technology on clinical trials and medical practices assesses the challenges and future prospects for incorporating genomic technologies into standard clinical practice. In 2003, the Human Genome Project completed the sequence of all human genetic material, in addition to an essentially complete catalogue of all human genes. ${ }^{16}$ Since the practice of genomics relies on large-scale, comprehensive analyses of genes, this information will prove very valuable in speeding the implementation of genomics in clinical settings. The F.D.A. has also issued draft guidelines for the submission of genomic data in drug applications, signifying a potentially pivotal change in the use of genomics in drug development. The clinical genomics environment is ripe with opportunities for all players in the field, especially the pharmaceutical companies.

\subsection{Ethical hurdles}

While the potential for the development of customized, genotype-based therapies is scientifically and clinically attractive, it raises ethical concerns for the conduct of research with human subjects, particularly with respect to confidentiality, risk-benefit analysis, DNA-banking and pharmacoeconomic issues. There are serious potential risks for discrimination and loss-of-privacy, which need to be addressed to formulate a policy to prevent such possible harm. Thus, despite the obvious scientific value of using families in pharmacogenomic trials, such studies raise serious ethical concerns that ensue from the dynamics and social significance of the family. ${ }^{18}$ The six ethical issues of most concern are: (a) regulatory oversight, (b) confidentiality and privacy, (c) informed consent, (d) availability of drugs, (e) access, and (f) clinicians changing responsibilities. Also, pharmacogenomics evokes the concept of consumer satisfaction, and acts at the borderline of the realm of health, in areas such as the use of medication for improving social and working skills, physical appearance, etc. For instance, many sports scientists warn that current performance-enhancing drugs may be a thing of the past once pharmacogenomics are introduced (16).

\section{Ethical, Legal and Social implications of Human Genomics}

The pharmaceutical industry is showing increasing interest in pharmacogenomics; similarly, governments, regulatory and advisory bodies in some countries are demonstrating a greater attention to this promising area of research. Simultaneously there is a similar growth in the understanding and ability to appropriately address the ethical, legal and social implications of pharmacogenomics. It is a field in its infancy; while evidence to data suggests reason for optimism, so far there are few applications in practice. In particular there is a lack of evidence on its impact in the context of developing countries. The complexity of human responses to medicines makes it cautious not to overestimate the probable impact of pharmacogenomics.

It is important to consider the extent to which pharmacogenomics might contribute to the further marginalization of individual's whose conditions and genetic peculiarities put them in the minority of patient populations. On the basis of genetic criteria, some conditions may be neglected as drug manufacturer's focus on developing those therapies that impact on the greatest proportion of the population. Broad pharmacogenomics programmes may require obtaining extensive genetic information, which raises concerns about the appropriate 
protection of patient's privacy and confidentiality. Moreover, the targeting of specific populations may result in the unfair discrimination against some groups. For instance, pharmacogenomic knowledge could be linked with specific racial or ethnic indicators, making it tempting to assume a biological linkage between race and responsiveness to particular medications, which could lead to inappropriate decisions about treatment.

Pharmacogenomics will need to be carefully evaluated by countries to determine its effectiveness, including its cost-effectiveness, compared to existing public health measures. It will also be important, if pharmacogenomics is to be effectively integrated into clinical practice, to educate both the public and health professionals about its basic principles, its benefits and limitations. Because much of the information provided by pharmacogenomic tests will be probabilistic, effective communication between physicians and patients about risk will be crucial to ensure informed discussion about appropriate treatment decisions. Its integration into clinical medicine needs to take into account questions of equity and fairness, and should be accompanied by appropriate regulatory structures that ensure that patient's privacy and confidentiality is protected $(17,18)$.

\section{Success so Far}

It is well known that genetic variability may alter drug catabolism (i.e. dihydropyrimidine dehydrogenase for 5-fluorouracil, thiopurine-S-methyl transferase for thiopurines, aldehyde dehydrogenase for cyclophosphamide) and anabolism (i.e. thymidine phosphorylase for capecitabine, deoxycitidine kinase for gemcitabine). Moreover, increased expression of transporter systems (i.e. the ATP binding cassette (ABC) superfamily) is associated with reduction of the cytoplasmic levels of drugs which may be unable to exert a cytotoxic effect. Additional systems could protect tumor cells from drug cytotoxicity, including the DNA repair machinery (nucleotide excision repair (NER) and DNA alkyltransferases) and antiapoptotic systems (i.e. bcl-2). Finally, alterations of drug targets may be associated with a decrease in the effectiveness of chemotherapy (i.e. mutations affecting tubulin and topoisomerase I for taxanes and irinotecan, respectively, and increased expression of thymidilate synthase for 5-fluorouracil). Therefore, genetic analysis has the potential to predict treatment efficacy and tolerability (19).

\subsection{Oncogenomics}

There are promising areas of cancer investigation which may represent the future scenario for therapeutic intervention, increasing treatment efficacy and/or reducing drug toxicity on the basis of genetic profile of cancer patient The identification of candidate genes on which pharmacogenetic analysis has to be focused is a complex process, mainly because the majority of anticancer drugs need to undergo an activating metabolism or are substrates of inactivating enzymes or excretion systems. Moreover, some factors which were considered to be predictors of cancer susceptibility to the therapy have gained a particular role in pharmacogenetics because of their role as indirect determinants of drug effects (i.e. p53 as a factor which triggers the apoptosis cascade in response to DNA damage). Finally, the existence of enzymatic systems which are involved in the repair of drug-induced damage (i.e. the activity of ERCC1 against alkylating agents) increases the number of genes which have to be investigated in order to predict the clinical outcome of chemotherapy. Therefore, the pharmacogenetic analysis should take into consideration both the molecular targets of anti-neoplastic agents and factors involved in drug disposition (20). Numerous gene variants frequently found in human populations may influence different stages of the neoplastic growth. These variants act through their products involved in various regulatory systems and metabolic chains at different levels of biological organization. Proliferation, differentiation and death of transformed and even malignant cells can be affected by pre-existing polymorphisms in genes exerting regulation of these basic processes (21).

Colorectal cancer (CRC) is the second most common cause of cancer death in Europe and the United States.5-Fluorouracil (5-FU)-based chemotherapeutic regimens are the standard treatment for the patients suffering from colorectal cancer. However, response rates for 5-FU as a single first-line treatment in advanced $\mathrm{CRC}$ are very poor. Combining 5-FU with the newer chemotherapies irinotecan (CPT-11) and oxaliplatin has improved response rates for advanced CRC (22). In the treatment of advanced colorectal cancer, irinotecan has become one of the most important drugs, despite its adverse effects (diarrhea and neutropenia) in some patients. Individual variation in response to irinotecan can be analyzed by using the data pharmacogenetics and pharmacogenomics would provide regarding the role of genetic polymorphisms in the main enzyme-systems in the metabolic pathways of irinotecan (23). 
Resistance to chemotherapy limits the effectiveness of current cancer therapies, including those used to treat colorectal cancer. Drug resistance can be intrinsic or acquired during treatment and is believed to cause treatment failure in a large percentage of patients with metastatic cancer. Furthermore, drug resistant micro metastatic tumour cells are also likely to reduce the effectiveness of adjuvant chemotherapy following surgery. Overcoming drug resistance is one of the main challenges of current cancer. Pharmacogenomics research could, however, identify patients most likely to benefit from chemotherapy and to identify which chemotherapy regimen to use.

Pharmacogenomics, especially, with increased number of the biomarkers used in the study, would enable tumour samples to be profiled on a global scale. Pre-clinical and clinical studies have already shown that combining information from more than one molecular biomarker increases our ability to predict tumor drug response. As well as analyzing the molecular phenotype of the tumor, pharmacogenomic and pharmacogenetic profiling of normal tissue may also be useful in predicting systemic toxicity. Individualization of therapy according to the molecular phenotype of tumor and patient should dramatically increase the effectiveness of chemotherapy (24). Investigation of common gene polymorphisms with regard to cancer progression and prognosis is still going through its initial stages. Its ultimate goal is, however, to reach a much higher level of understanding the forces driving the process of malignant growth. This understanding would allow predicting its future course in each individual case and developing individually adopted means of its elimination or containment.

Genomic abnormalities in lung cancer suggest that the application of a pharmacogenomic approach has the potential to greatly improve survival in certain subpopulations of patients with non-small cell lung cancer (NSCLC). The studies further led to the conclusion that ribonucleotide reductase M1 polypeptide (RRM1) is a biologically and clinically important determinant of malignant behavior in NSCLC and represents a strong predictor of outcome in patients with resectable disease. Subgroups of NSCLC patients that might have different responses to treatment are primarily defined on the basis of clinical parameters such as performance status, personal preference, convenience, central nervous system metastases, histology, bleeding disorders, gender and smoking status. However, pharmacogenomics has the potential to allow the selection of specific patients on a genetic basis. It is hypothesized that this specific tailoring of therapy, guided by individual patient genetics, could lead to unequivocally superior responses following chemotherapy treatment (25).

Functions of single genes are typically studied by creating a mutation or deletion in a rodent model. However, as this historical approach is too time consuming and cost inefficient to be applied to every gene responsible for the characteristics of a single cancer, and it is unknown whether the results would be applicable to human cancer. Recently, DNA micro arrays have been used to profile and compare the global gene expression patterns of different cancers in human patients. Much work remains to be done before micro array technology can be applied to the general population. Although several groups have successfully narrowed the number of genes needed to determine the subgroup or prognosis of a tumor, the list is still too extensive to screen in every patient and pharmacogenetic analysis has the potential to predict treatment efficacy and tolerability. Another major problem encountered in pharmacogenetic and pharmacogenomic studies is the need for extensive validation of available technology. There is also considerable difficulty in obtaining a suitable amount of tissue from patients during the course of their disease and the extremely complex regulation of gene function. From this perspective, the evaluation of the cellular effect of drugs in relation to protein expression and function (pharmacoproteomics) may be able to overcome these obstacles and allow the optimization of cancer chemotherapy in association with a pharmacogenetic approach $(26,27)$.

\subsection{Cardiovascular Therapy}

Pharmacogenomics is markedly influencing the management of essential hypertension. While the final phenotype of elevated blood pressure may be similar in different patients, the underlying hereditary determinants of the increase in blood pressure levels are likely to be polygenic and heterogeneous (28). The identification of genetic markers of drug response will help to achieve a better control of blood pressure in the population, by allowing a better tailor of antihypertensive therapy to individual patients (29). Several polymorphisms in blood pressure-regulating drug receptors [e.g., beta-adrenergic receptors (ADBR)] and receptor response pathways [G-protein beta 3 subunit (GNB3), Renin-Angiotensin-Aldosterone System (RAAS)] have been predictive of responses to blood pressure lowering treatment. Genomic studies on the Renin- 
Angiotensin-Aldosterone System constitute the most information among the different antihypertensive drug classes (30).

Statins are widely prescribed and are established as first-line therapy for the primary and secondary prevention of coronary artery disease (CAD). Data from pharmacogenetic studies are expected to have great impact in statin therapy. The characteristics of the drugs and the pathophysiological mechanisms of adverse effects are understood from the results of pharmacogenetic and pharmacogenomic studies related to statin therapy. Also, exploring genetic variation related to susceptibility to serious adverse events such as myositis, as well as the cost-effectiveness of statin therapy potentially would be of great value. It may be easier to elucidate the genetic variability responsible for variations in drug response than the genetic variabilities responsible for common chronic diseases such as CAD. Data from pharmacogenetic studies are expected to have great impact in the clinical arena in the future and will allow for the identification of persons likely to get the greatest and least benefit from a given intervention $(31,32)$

The prevention of cardiovascular disease is critically dependent on lipid-lowering therapy and although, these drugs are generally well tolerated, severe adverse effects can occur in a minority of patients. Furthermore, a subset of patients does not respond to cholesterol-lowering therapy with a reduction in coronary heart disease progression. The influence of ethnicity on pharmacogenetic responses must be taken into account by performing and comparing clinical trials in various ethnic groups. In the near future, pharmacogenomics may allow the identification of patient subgroups which most likely profit from lipid-lowering therapy. Furthermore, genotyping of high-risk patients may prevent adverse effects in individuals with genetic predisposition (33).

In cardiovascular disease, oral anticoagulants, non-steroidal anti-inflammatory drugs, oral hypoglycemic drugs and other drugs are affected by genetic polymorphisms of the cytochrome P450 drug metabolizing enzyme. Pharmacogenomic studies in patients or healthy volunteers reveal up to 10-fold differences in pharmacokinetic parameters due to genetic polymorphisms. Pharmacogenetics based dose adjustments are one tool to individualize drug treatment according to genetic factors (34).

Warfarin is a commonly prescribed oral anticoagulant for the treatment and prevention of thrombotic diseases. However, warfarin has a narrow therapeutic range and there is greater than 10 -fold inter-individual variability in the dose required to attain a therapeutic response. An insufficient dose may fail to prevent thromboembolism, while an overdose increases the risk of bleeding. Warfarin therapy management is challenging for several reasons including the need to determine a safe and effective maintenance dose during the early phase of therapy and the fact that maintenance doses must be adjusted to compensate for changes in patient's weight, diet, disease state, concomitant use of other medications, and genetic factors. It takes several weeks to achieve a stable warfarin control, though the risks of overdose and increasing the warfarin induction period still remain. However, recent research has suggested that genetic variation in cytochrome enzymes greatly influences effective warfarin dose. Pharmacogenetic and pharmacogenomic algorithms could potentially minimize the risk of overdose during warfarin induction (35-37)

\subsection{Central Nervous System (CNS) related conditions}

There are ample evidences to suggest that genetic changes in neurotransmitter systems may be important in the pathogenesis of variety of CNS disorders like Alzheimer's disease, Schizophrenia and Epilepsy. Pharmacotherapy of Alzheimer's disease (AD) has attracted the interest of both the public and scientific world. The more the enigma of AD unravels, the clearer it seems that a mechanism-based approach to therapy may be a more practical way to disease-modifying discoveries to manage this devastating disease. Pharmacogenomics has a potential to contribute invaluably to the proposed mechanism-based approach (i.e. genotype-based approach) by providing answers not only for familial AD but also for non-familial (sporadic) $\mathrm{AD}(38)$.

Although antipsychotic drugs are effective in alleviating schizophrenic symptoms, individual differences in patient response suggest that genetic components play a major role, and pharmacogenetic studies have indicated the possibility for a more individually based pharmacotherapy. Pharmacogenomics aids in understanding how genetics influence disease development and drug response and contribute to discovery of new treatments. Prospective genotyping of schizophrenic patients for the many genes at the level of the drug target, drug metabolism, and disease pathways will contribute to individualized therapy matching the patient's unique genetic make-up with an optimally effective drug (39). 
There has been very little change in the incidence of drug resistant epilepsy despite the introduction of several new drugs in the last decade. It is becoming increasingly clear that genetic polymorphisms play an integral role in variability in both antiepileptic drug pharmacokinetics and pharmacodynamics. The publication of the human genome and increasing sophisticated and powerful genetic tools offers new methods for screening drugs and predicting deadly idiosyncratic side effects. Studies of genetic variation that relate to proteins involved in antiepileptic drug kinetics and dynamics will identify key polymorphisms in the endogenous molecules that determine degrees of drug efficacy and toxicity. Delineation of these effects in the coming years will promote enhanced success in the treatment of epilepsy (40-42).

\subsection{Respiratory conditions}

Pharmacogenetic studies of drugs used in the treatment of asthma have produced a few examples of reduced response in patients carrying specific genotypes in genes. Genotyping for individual pharmacogenetic responses may be useful in establishing an anti-asthmatic therapy. Future objectives include expanded gene knowledge from asthma genetic and genomic studies, the development of new preventive and curative treatments and the construction of asthma functional pharmacogenomic profiles. It is likely that in the near future, information regarding individual genetic polymorphisms will allow us to identify those patients most likely to respond, those likely to experience adverse effects from therapeutic interventions in asthma $(43,44)$.

\subsection{Pharmacogenomics in diagnosis}

Single nucleotide polymorphisms (SNPs) within genes affect drug disposition or drug targets. One of the well-characterized examples is highly relevant to inflammatory bowel disease therapeutics, that of thiopurine methyltransferase pharmacogenetics. By using high-throughput SNP genotyping, combined with careful phenotypic characterization of disease, previous term pharmacogenomic next term research carries the potential of identifying individual biomarkers that predict the relative likelihood of benefit or risk from a therapeutic intervention.

\subsection{Miscellaneous}

Pharmacogenomics shows promising result in various other areas of therapeutics viz. Immunosuppressive therapy for organ transplant and autoimmune diseases, genetic polymorphism of CYP 450 enzymes and recently in the pharmacotherapy of HIV-infection $(43,44)$.

Pharmacogenomics holds many promises for improved treatment of a large variety of medical conditions, including immunosuppression for organ transplantation and autoimmune disease. The enormous potential of the human genome, if deciphered, can be successfully used within the pharmacogenomic framework for individualizing immunosuppressive therapy based on the patient's genomic profile.

Pharmacogenomics in epidermal growth factor receptor (EGFR) tyrosine kinase inhibitors has made it clear that some patients benefit from EGFR therapies and these differential responses are mostly due to the genetic nature.

\subsection{Pharmacogenomic in clinical trial design:}

The clinical practice of evidence-based medicine concept derived from the fundamental principal of clinical trial. The concept of trial design allows a meaningful interpretation of the data include sufficient sample size, statistical power and control of sampling bias. Clinical trials are usually classified according to five phases namely phase-o phase-1, phase-II, phase-III and phase-IV. Generally, the randomized clinical trial (RCT) and, in particular, the double-blind RCT is considered the 'gold standard' in determining treatment efficacies. Indeed, the objective of randomization is to reduce bias and variability as much as possible pharmacogenomic profiling is generally incorporated into trials either retrospectively or prospectively (45).

Retrospective studies use genotyping and high throughput-sequencing techniques to identify genotypes and attempt to correlate them with PHENOTYPES, such as disease or drug responsiveness. DNA libraries are now being established and archived for future research. The use of pharmacogenomic profiling in prospective studies is focused on attempts to reduce pharmacokinetic variability or the incidence of adverse events. 
Typically, this consists of stratifying research subjects or excluding certain subjects from the trial on the basis of genotype. The example of some genotyping incorporation of the trial is given in Table-1

Table - 1: Example of genotyping at the different stages of the trial

\begin{tabular}{|l|l|}
\hline Genotyping at different stages of clinical trials: \\
\hline Phase I & $\begin{array}{l}\text { - Genotyping might be carried out to try and identify and correlate } \\
\text { polymorphisms with phenotypic elements } \\
\text { (such as pharmacokinetic/pharmacodynamic properties, excretion and serum } \\
\text { levels) } \\
\text { • Certain research subjects with particular genotypes might be excluded from } \\
\text { the trial } \\
\text { • Certain research subjects with particular genotypes might be enrolled } \\
\text { preferentially }\end{array}$ \\
\hline Phase II & $\begin{array}{l}\text { - Genotyping might be used for genotype-phenotype correlations } \\
\text { with differences in efficacy using the } \\
\text { candidate-gene approach }\end{array}$ \\
\hline Phase III & $\begin{array}{l}\text { Use results from Phase I and II trials to design an optimal large-scale Phase III } \\
\text { study } \\
\text { - Test candidate genes for efficacy and metabolism } \\
\text { - Might be useful to conduct large-scale genotyping to discover new } \\
\text { pharmacogenomic markers } \\
\text { - Identify which sub-populations show more adverse events to certain drugs } \\
\text { - Identify responders versus non-responders to certain drugs }\end{array}$ \\
\hline $\begin{array}{l}\text { Studies to assess the following: } \\
\text { Pare adverse events and the relationship of such events to specific sub- } \\
\text { populations } \\
\text { • Marketing considerations; that is, whether a diagnostic test might be capable } \\
\text { of distinguishing the drug of } \\
\text { interest from competitors, and whether the market for a given drug would } \\
\text { justify the development of } \\
\text { diagnostic testing }\end{array}$ \\
\hline
\end{tabular}

Successful pharmacogenomic study example is given in Table- 2 which potentiate the drug discovery at minimal cost by predicting the prior toxicity and efficacy profile of the drug.

Table- 2: Examples of successful clinical pharmacogenomic studies

\begin{tabular}{|l|l|l|l|l|}
\hline $\begin{array}{l}\text { Clinical } \\
\text { condition or } \\
\text { disease }\end{array}$ & Drug(s) & $\begin{array}{l}\text { Genetic } \\
\text { polymorphism(s) }\end{array}$ & Outcome & References \\
\hline $\begin{array}{l}\text { Alzheimer's } \\
\text { disease }\end{array}$ & Tacrine & APOE4 & $\begin{array}{l}\text { APOE4 homozygotes } \\
\text { have poor responses } \\
\text { compared with research } \\
\text { subjects with other APOE } \\
\text { alleles }\end{array}$ & $(46)$ \\
\hline Schizophrenia & Clozapine & $\begin{array}{l}\text { 5-HT2A receptor } \\
\text { C102allele }\end{array}$ & $\begin{array}{l}\text { C102 homozygotes seem } \\
\text { to respond better to the } \\
\text { atypical antipsychotic } \\
\text { clozapine }\end{array}$ & (47-49) \\
\hline $\begin{array}{l}\text { Coronary } \\
\text { atherosclerosis }\end{array}$ & Pravastatin & $\begin{array}{l}\text { Cholesteryl-ester } \\
\text { transfer protein } \\
\text { (CETP) with }\end{array}$ & $\begin{array}{l}\text { B1B1 homozygotes have } \\
\text { better response to } \\
\text { pravastatin then either }\end{array}$ & $(50)$ \\
\hline
\end{tabular}




\begin{tabular}{|c|c|c|c|c|}
\hline & & $\begin{array}{l}\text { polymorphisms at } \\
\text { the TaqB1 site } \\
\text { (alleles B1 and } \\
\text { B2); lipoprotein } \\
\text { lipase (LDL); and } \\
\beta \text {-fibrinogen }\end{array}$ & $\begin{array}{l}\text { B1B2 heterozygotes or } \\
\text { B2B2 homozygotes }\end{array}$ & \\
\hline Gastric ulcers & $\begin{array}{l}\text { Omeprazole } \\
\text { and } \\
\text { amoxicillin }\end{array}$ & CYP2C19 & $\begin{array}{l}\text { CYP2C19 poor } \\
\text { metabolizers responded } \\
\text { more favorably to dual } \\
\text { therapy than subjects with } \\
\text { extensive metabolizer } \\
\text { genotypes }\end{array}$ & (51) \\
\hline Asthma & $\begin{array}{l}\text { Zileuton and } \\
\text { Montelukast }\end{array}$ & $\begin{array}{l}\text { ALOX5 genotype } \\
\text { CYP2D6 }\end{array}$ & $\begin{array}{l}\text { Reduced response among } \\
\text { heterozygotes }\end{array}$ & (52) \\
\hline
\end{tabular}

ALOX5- arachidonate 5-lipoxygenase; APOE4-apolipoprotein E4 allele.

\section{Conclusion}

Pharmacogenomics unequivocally has an increasingly important role in drug discovery and development. Pharmacogenomics can potentially lead to better clinical candidates, fewer clinical failures, faster drug development and breakthrough medicine. While it is believed that pharmacogenomics is already delivering these promises, there is still a long way to go and genomics should not be thought of in isolation, it should be used alongside other disciplines by different types of institutions to tackle drug discovery as a multidisciplinary enterprise. However, though pharmacogenomics offer new approaches to an improved healthcare and to targeted care, it is not cost effective and it remains to be seen how the pharmaceutical industry will assess the risk-benefit of such technologies

\section{References}

1. Grant S.F.A. Pharmacogenetics and pharmacogenomics: tailored drug therapy for the 21 st century. Trends Pharmacol. Sci., 2001, 22(1), 3-4.

2. Katrina M Romagnoli, Richard D Boyce, Philip E Empey, Yifan Ning, Solomon Adams, Harry Hochheiser. Design and evaluation of a pharmacogenomics information resource for pharmacists. J Am Med Inform Assoc., July 2017,24(4), 822-31

3. Katara P. Role of bioinformatics and pharmacogenomics in drug discovery and development process. New Model Anal Health Inform Bioinforma., 2013 2, 225.

4. Hughes J.E. Genomic technologies in drug discovery and development. DDT, 1999, 4(1), 6.

5. Personalized Medicine - Scientific and Commercial Aspects, Jain Pharma Biotech, Feb 2016

6. Daniel W.N., Elliot S.V. Advances in pharmacogenomics and individualized drug therapy: exciting challenges that lie ahead. Eur. J. Pharmacol. 2004, 500, 267-80.

7. Personalized Medicine: The Impact of Pharmacogenomics on Pharmaceutical R\&D and Sales, Datamonitor, Aug 2002.

8. Klein M.E., Parvez M.M., Shin J.G. Clinical Implementation of Pharmacogenomics for Personalized Precision Medicine: Barriers and Solutions. J Pharm Sci., Volume 2017,106(9), 2368-79.

9. Evans W.E., Rolling M.V. Moving towards individualized medicine with pharmacogenomics. Nature., 2004, 429, 464-68.

10. Roses A.D. How will pharmacogenetics impact the future of research and development? DDT, 2001, 6(2), 59-60.

11. Pirazzoli A, Recchia G., Pharmacogenetics and pharmacogenomics: are they still promising? Pharmacol. Res. 2004, 49, 357-61.

12. Peet N.P., Bay P. Pharmacogenomics: challenges and Opportunities. DDT 2001 6(10): 495-498

13. Johnson A.D., Wang DW Sadee. Polymorphisms affecting gene regulation and mRNA processing: Broad implications for pharmacogenetics, PHARMACOL THERAPEUT 2005,106, 19-38

14. Amouyel P. A critical assessment of the role of pharmacogenomics and pharmacogenetics approaches to cardiovascular diseases, Pharmacogenomics J., 2001, Volume 1(2), 95-7. 
15. The Role of Pharmacogenomics in Personalized Medicine, Decision Resources, Inc., June 2006

16. Clinical Genomics: The Impact of Genomic Technology on Clinical Trials and Medical Practice, CHA Advances Reports, Jan 2004

17. Amalia M.I. Ethical considerations in clinical pharmacogenomics research, TIPS, 2000; 21.

18. Buchanan A, Califano A, Kahn J, McPherson E, Robertson J, Brody B. et al, Pharmacogenetics: ethical issues and policy options, Kennedy Institute of Ethics J 2002, 12(1), 1-15.

19. Mafalda M.D, Krupa Depala, Helena M.W, Michael B.W, Michael J.S, Claire Anderson, Ross A. McKinnon. Reforming Pharmaceutical Education to Enhance the Global Uptake of Pharmacogenomics and Personalized Medicine. Curr Pharmacogenomics Person Med., 10 ,3, 2012.

20. Yagil Y, Yagil C. Insights into pharmacogenomics and its impact upon immunosuppressive therapy, Transpl Immunol., 2002, 9, 203-9.

21. Di Paolo, Antonello. Pharmacogenetics of neoplastic diseases: new trends. Pharmacological Research, 2004, 49, 331-42.

22. Loktionov A. Common gene polymorphisms, cancer progression and prognosis, Cancer Letters. 2004, 208, 1-33.

23. Longley D, Allen W.L, Johnston P.G. Drug resistance, predictive markers and pharmacogenomics in colorectal cancer, Biochim Biophys Acta. 2006, 1766(2): 184-96.

24. de Jong F.A., de Jonge M.A, Verweij J, Mathijssen R.H. Role of pharmacogenetics in irinotecan therapy, Cancer Letters, 2006, 234, 90-106.

25. Bepler G. Pharmacogenomics: A reality or still a promise? Lung Cancer, 2006, 54, S3-S7

26. John R.S. Michael D.K. Exploring the Human Genome in Cancer with Genomic Approaches. J Vasc Interv Radiol., 2006, 17(8), 1225-33.

27. Israel E, Genetics and the variability of treatment response in asthma. J. Allergy Clin. Immunol., 2005, 115(4)1, S532-38

28. Sharma P, Fatibene J, Ferraro F, Jia H, Monteith S, Brown C et al, A genome-wide search for susceptibility loci to human essential hypertension. Hypertension, 2000, 35, 1291-96.

29. Allison B.C., Daniel W.C. Precision medicine: from pharmacogenomics to pharmacoproteomics. Clinical Proteomics, 2016, 25, 13.

30. Trotta R, Donati M.B, Iacoviello L. Trends in pharmacogenomics of drugs acting on hypertension. Pharmacol Res., 2004, 49, 351-56.

31. Arnett D.K., Claas S.A., Glasser S. P. Pharmacogenetics of antihypertensive treatment, Vascul Pharmacol 2006, 44, 107-18.

32. Kajinami K, Akao H, Polisecki E. Schaefer E.J. Pharmacogenomics of Statin Responsiveness. Am J Cardiol., 2005, 96(9), 65-70.

33. Roden D.M., Van Driest S.L., Wells Q.S., Mosley J.D., Denny J.C., Peterson J.F. Opportunities and Challenges in Cardiovascular Pharmacogenomics From Discovery to Implementation. Circ Res 2018, 122, 1176-90.

34. Schmitz G., Langmann T. Pharmacogenomics of cholesterol-lowering therapy, Vasc Pharmacol.2006, 44(2), 75-89.

35. Kirchheiner J, Seeringer A. Clinical implications of pharmacogenetics of cytochrome P450 drug metabolizing enzymes. Biochim et Biophys Acta 2007, 1770, 489-94.

36. Yin T, Miyata T. Warfarin dose and the pharmacogenomics next term of CYP2C9 and VKORC1 Rationale and perspectives. Thromb Res 2007, 120(1), 1-10.

37. Crentsil V. The pharmacogenomics of Alzheimer's disease, Ageing Res Rev. 2004, 3, 153-69.

38. Rigaud A.S., Traykov L, L. Caputo ,Guelfi M.C., Latour F, Couderc R, Moulin F, J. De Rotrou , Forette F, Boller F. The apolipoprotein E4 allele and the response to tacrine therapy in Alzheimer's disease. Eur. J. Neurol. 2000, 7, 255-258.

39. Kawanishi H, Tachibana T, Suzuki et al, Pharmacogenomics and schizophrenia. Eur. J. Pharmacol, 2000, 410, 227-41.

40. Gregory L.H. The interface of preclinical evaluation with clinical testing of antiepileptic drugs: role of pharmacogenomics and pharmacogenetics. Epilepsy Res. 2002, 50, 41-54.

41. Thomas N.F., Russell J.B. The relationship between the pharmacology of antiepileptic drugs and human gene variation: An overview, Epilepsy Behav 2005, 7, 18-36.

42. Franco Pignatti P. Trends in pharmacogenomics of drugs used in the treatment of asthma. Pharmacol Res, 2004, 49, 343-9. 
43. Davis J.S., Weiss S.T., Tantisira K.G. Asthma Pharmacogenomics: 2015 Update. Curr Allergy Asthma Rep. 2015, Jul, 15(7), 42.

44. Ishizuka T. CYP2C pharmacogenetics and tailor-made therapeutic implications. International Congress Series 2002, 1244, 1-9.

45. Tim RC, Lallemant M. Pharmacogenetics of antiretroviral drugs for the treatment of HIV-infected patients:An update, Infection, Genetics and Evolution 2007, 7(2), 333-42.

46. Jimeno A, Hidalgo M. Pharmacogenomics of epidermal growth factor receptor (EGFR) tyrosine kinase inhibitors. Biochim et Biophys Acta. 2006, 1766(2), 217-29.

47. Poirier J, Delisle M.C., Quirion R, Aubert I, Farlow M, Lahiri D, Hui S, Bertrand P, Nalbantoglu J, Gilfix B.M. Apolipoprotein E4 allele as a predictor of cholinergic deficits and treatment outcome in Alzheimer disease. Proc. Natl Acad. Sci. USA 1995, 92(26), 12260-4.

48. Solomon M.A., Karryn R.C., Philip E.E. Clinical hargenomics. CJASN. 2018, 13(10), 1561-71.

49. Richard F, Helbecque N, Neuman E, Guez D, Levy R, Amouyel P. APOE genotyping and response to drug treatment in Alzheimer's disease. Lancet. 1997, 349, 539-40.

50. Arranz M, Collier D, Sodhi M, Ball D, Roberts G, Price J, Sham P, Kerwin R. Association between clozapine response and allelic variation in 5-HT2A receptor gene. Lancet. 1995, 346, 281-82.

51. Joober R, Benkelfat C, Brisebois K, Toulouse A, Turecki G, Lal S, Bloom D, Labelle A, Lalonde P, Fortin D, Alda M, Palmour R, and Rouleau G.A. T102C polymorphism in the 5HT2A gene and schizophrenia: relation to phenotype and drug response variability. J. Psych. Neurosci. 1999, 24, 141146.

52. Masellis M, Basile V, Meltzer H.Y., Lieberman J.A., Sevy S, Macciardi F.M., Cola P, Howard A, Badri F, Nöthen M.M., Kalow W, Kennedy J.L. Serotonin subtype 2 receptor genes and clinical response to clozapine in schizophrenia patients. Neuropsychopharmacol. 1998, 19, 123-32.

53. Kuivenhoven J.A., Jukema J.W., Zwinderman A.H., de Knijff P, McPherson R, Bruschke A.V., Lie K.I., Kastelein J.J. The role of a common variant of the cholesteryl ester transfer protein gene in the progression of coronary atherosclerosis. New Engl. J. Med. 1998, 338, 86-93.

54. Furuta T, Shirai N, Takashima M, Xiao F, Hanai H, Sugimura H, Ohashi K, Ishizaki T, Kaneko E. Effect of genotypic differences in CYP2C19 on cure rates for Helicobacter pylori infection by triple therapy with a proton pump inhibitor, amoxicillin, and clarithromycin. Clin. Pharmacol. Ther 2001, 69, $158-68$.

55. Drazen J.M., Yandava C.N., Dubé L, Szczerback N, Hippensteel R, Pillari A, Israel E, Schork N, Silverman E.S., Katz D.A., Drajesk J. Pharmacogenetic association between ALOX5 promoter genotype and the response to anti-asthma treatment. Nature Genet. 1999, 22, 168-170. 